\title{
Handbook of Early Christianity. Social Science Approaches
}

Walnut Creek, Altamira Press, 2002, 802 p.

\section{Anna Van den Kerchove}

\section{(2) OpenEdition}

\section{Journals}

\section{Édition électronique}

URL : http://journals.openedition.org/assr/5412

DOI : $10.4000 /$ assr.5412

ISSN : $1777-5825$

\section{Éditeur}

Éditions de l'EHESS

\section{Édition imprimée}

Date de publication : 1 juin 2007

ISBN : 978-2-7132-2143-9

ISSN : 0335-5985

\section{Référence électronique}

Anna Van den Kerchove, "Handbook of Early Christianity. Social Science Approaches », Archives de sciences sociales des religions [En ligne], 138 | avril - juin 2007, document 138-9, mis en ligne le 29 novembre 2013, consulté le 21 septembre 2020. URL : http://journals.openedition.org/assr/5412 ; DOI : https://doi.org/10.4000/assr.5412

Ce document a été généré automatiquement le 21 septembre 2020.

(c) Archives de sciences sociales des religions 


\title{
Handbook of Early Christianity. Social Science Approaches
}

Walnut Creek, Altamira Press, 2002, 802 p.

\author{
Anna Van den Kerchove
}

1 Avec cet imposant volume sur les premiers temps du christianisme, les éditeurs nous offre un guide du christianisme passionnant. Ils ont réuni vingt-sept articles, assez longs, qui abordent les premiers temps du christianisme dans une perspective sociale. Il ne s'agit pas d'une histoire sociale mais d'une sociohistoire. La nuance est extrêmement importante : les auteurs ont abordé le christianisme primitif avec des approches issues des sciences sociales. L'ensemble des articles est réparti en six parties thématiques, et le tout est complété par près de soixante pages de références, cinquante pages bibliographiques (dues à J. Duhaime) et trois index : auteurs, références bibliques et thèmes.

2 Il est assez difficile de rendre compte d'un ouvrage aussi dense et riche. La première partie aborde les perspectives générales de l'approche sociale du christianisme primitif, posant le cadre pour les parties suivantes, avec trois articles complémentaires, historiographiques et méthodologiques. D.G. Horrell débute le volume en décrivant le développement moderne de la sociologie, en relation avec son contexte historique, et les différentes approches des études sur le christianisme et le Nouveau Testament. Paul-André Turcotte apporte un complément, en exposant les grandes théories sociologiques, qu'il organise autour de grandes thématiques, comme le conflit social ou le charisme prophétique. Quant à Anthony J. Blasi, il développe les perspectives méthodologiques qu'il faut adopter dans l'étude des premiers temps du christianisme, rappelant que les principales sources sont essentiellement littéraires, ce qui implique d'être attentif aux auteurs et aux destinataires et d'avoir une solide méthodologie pour les approcher au mieux.

3 Dans la deuxième section, les auteurs se concentrent chacun sur une méthode particulière. Dans un premier temps, Carolyn Osiek aborde le problème de l'interprétation sociale de la documentation archéologique et architecturale, notamment la manière dont les groupes religieux adaptent l'architecture à leur besoin. 
L'auteure termine en expliquant le déroulement de certaines scènes évangéliques à l'aide du social. Ritva $\mathrm{H}$. Williams se consacre à la figure de Jésus, notamment à partir de Mathieu I,1-25, pour montrer comment on peut mener une enquête historique à partir de textes évangéliques. Steven L. Bridge reste dans la même veine : il s'intéresse aux sources de textes et donne un catalogue très utile de critères méthodologiques pour analyser les sources, agrémenté de nombreux exemples évangéliques. Il se tourne ensuite vers la critique rédactionnelle, la manière dont l'auteur utilise les sources qui sont à sa disposition, et il applique cela aux évangiles de Mathieu et de Marc. L'article de Robert $\mathrm{A}$. Wortham est particulièrement novateur : l'auteur démontre l'intérêt que peut avoir une analyse statistique des textes. Il en détaille les bases et les principes, avant de donner un exemple d'application avec le prologue de Jean. Ernst R. Wendland présente, quant à lui, les apports de l'analyse rhétorique, avec les différents types de rhétorique existant, pour une meilleure compréhension des textes néo-testamentaires. Peter Staples aborde, pour sa part, la méthode structuraliste, afin d'étudier et de comprendre la conception de l'espace et du temps sociaux du Second Temple, en relation avec les pratiques rituelles.

4 La troisième partie présente une série d'articles sur le contexte et l'émergence du mouvement de Jésus et du christianisme primitif. Frederik Bird étudie le christianisme primitif comme un mouvement missionnaire et diversifié, en l'abordant de la même manière que les nouveaux mouvements religieux à d'autres époques. Peter Richardson et Douglas Edwards étudient, en termes socio-historiques, les correspondances entre le mouvement de Jésus et le mouvement de protestation sociale palestinien. S'aidant des Évangiles et relevant des parallèles avec d'autres sources, les auteurs pensent que le mouvement de Jésus fait partie du mouvement de protestation sociale palestinien. Les deux articles suivants s'intéressent aux contacts culturels en relation avec le christianisme primitif. Donald A. Lielsen sélectionne quelques points de rencontre pour étudier leur rôle dans le développement du judaïsme tardif et du christianisme primitif. Anthony J. Blasi aborde la culture chrétienne en termes d'interaction, ce qui permet, selon lui, de mieux comprendre les phénomènes socioculturels du christianisme. Les trois derniers articles de cette partie tournent autour de la formation de l'identité chrétienne. David G. Horrell s'intéresse au fait de devenir chrétien et à la construction de l'identité chrétienne. Il a recours, pour cela, aux ressources des sciences sociales, comme la théorie de la structuration, l'identité sociale et le conflit, pour les appliquer diachroniquement à différentes époques du christianisme depuis l'époque de Jésus jusqu'aux années 100. H.Cl. Kee aborde le rôle de leadership et la formation de la communauté, tandis que Jack T. Sanders analyse la distance sociale entre, d'une part, les chrétiens et, d'autre part, les païens et les juifs.

5 La quatrième partie est consacrée au pouvoir, à l'inégalité et aux différences. Philipp A. Harland étudie la nature des connections entre les chrétiens et l'élite, alors que John W. Marshall et Russell Martin replacent le développement du christianisme primitif dans le cadre institutionnel de l'époque, en s'intéressant au gouvernement et à la loi publique. Les deux articles suivants approfondissent les relations avec l'Empire romain. Le premier, dû à Harold Remus, s'intéresse au phénomène de la persécution et à ses conséquences quant aux différentes attitudes adoptées face à elle et aux conceptions diverses de l'identité. Le second article, de Warren Carter, part de l'idée que le christianisme constituait un défi pour l'Empire romain pour en venir à considérer les textes chrétiens et les communautés comme résistance. Les trois derniers articles abordent plutôt l'aspect économique du christianisme primitif. Nicola Denzey examine 
les limites des catégories ethniques. Philipp A. Harland fait un état historiographique de la question de l'économie de la Palestine du premier siècle après J.-C., avant d'en venir aux problèmes méthodologiques et de donner les directions futures de la recherche. Dimitris J. Kyrtatas, pour sa part, étudie les modes et les relations de production, chez les chrétiens, en les replaçant dans le contexte de l'époque. David A. Fiensy aborde la question de ce que les chrétiens avaient le droit de faire ou de ne pas faire comme travail pour vivre.

6 La dernière partie est consacrée aux approches et aux phénomènes psychosociaux. Nicholas $\mathrm{H}$. Taylor analyse, à partir du cas de Paul, les différents types d'identité. Richard K. Fenn porte un regard psychanalytique sur les conflits fratricides, en partant de l'étude de Hyam Maccoby sur Judas comme frère de Jésus. Jack T. Sanders conclut cet ouvrage avec un article sur la conversion, en particulier ses aspects sociologiques.

7 Ce guide, dont nous n'avons donné qu'un bref aperçu, s'avère être ainsi un véritable instrument de travail, original par les approches adoptées, par les méthodes exposées. Son utilisation sera très stimulante pour quiconque est intéressé par l'étude des débuts du christianisme. 